was oxygen, and pathogenic strains distinguish themselves by their ability for survival at low oxygen tensions. In the human lung most infections resulted in the embedding of bacilli in solid caseous foci in which they rapidly decreased. Rapid bacillary multiplication followed softening of caseum, presumably due to increased availability of oxygen, and possibly to the release of nutrients by proteolysis. It is the softening of caseum that is usually responsible for tuberculous disease as distinct from infection. Solid caseation followed by softening was not found in the hyperimmune animal injected with bacilli in adjuvant. Dr. Lack has shown that human plasmin can digest human caseum. The increased morbidity of females between 15 and 35 years could possibly be related to enhanced plasmin activity at the pre-menstrual period. Abnormal plasmin activation may also be the mechanism of the rapid cavitation following severe psychical trauma. The study of the factors of such mechanisms was the most fruitful approach to the problem of human tuberculosis.

Dr. E. Suter (University of Florida) described the effect of phagocytosis on the respiration of microorganisms. The important contribution of the phagocytic system to natural resistance can be mediated through either bactericidal substances (for example, phagocytin and leukin), or through subtle influences upon bacterial metabolism and on enzymatic breakdown of bacterial components. Using organisms labelled with carbon-14, the fate of bacillary carbon can be followed in the intracellular environment. Tubercle bacilli maintained their respiratory-rate within phagocytes. Furthermore, little or no bacillary carbon was handled by phagocytic enzymes and appeared in the respiratory carbon dioxide. This metabolic resistance of tubercle bacilli to the phagocytic environment seemed to depend upon their structural intactness. Thus sonic vibration of bacilli or the presence of non-ionic detergents altered this relationship to the advantage of the cell. The findings were quite different when organisms were phagocytosed which were less resistant to intra-cellular environment, such as Mycobacterium phlei, Bacillus subtilis, Staphylococcus albus and aureus, and Streptococcus pneumoniae. Their metabolic activity was reduced within one or two hours to one-tenth or onehundredth of their original activity, and bacillary carbon appeared very quickly in the respiratory carbon dioxide of the phagocytes.

Dr. D. Rowley (St. Mary's Hospital) described the work of J. G. Howard, A. C. Wardlaw and himself which derived from the observation, some years ago, that injection into mice of small quantities of cell walls of Gram-negative bacteria produced rapidly developing immunity to subsequent infections by other Gram-negative organisms. This non-specific immunity was of short duration (up to 14 days) and was not accompanied by any significant changes in the level of complement or natural antibody. Lipopolysaccharide from cell walls produced this immunity in doses as small as $1 \mu \mathrm{gm}$. per mouse, and more recently the lipid $A$ fraction of this has been found highly active. Although injected lipopolysaccharide rapidly increased the serum properdin, it is unlikely that this accounted for the immunity, for this extended to organisms not affected by the serum bactericidal activity of properdin and complement. The intravenous injection of lipopolysaccharide labelled with phosphorus-32 resulted in the rapid removal of most of this by the reticulo-endothelial system. Using the rate of removal of this labelled material as a test of function of the reticulo-endothelial system, it was shown that increased phagocytic activity accompanied the development of immunity.

Dr. D. G. Evans (Medical Research Council, Hampstead) discussed the immunity produced in mice by intraperitoneal injection of pertussis vaccine against the intracerebral challenge of living virulent organisms. The steep immune response without lag was unlike that associated with antibody production, and occurred before circulating specific antibodies were detectable. This early immunity was transient and declined at 10-14 days, to be followed by the slower development of a true antibody type of immunity, which reached maximum at 28 days. The early and later immunity were associated with a common factor so that vaccines with a good early response also elicited a good later response. The purified pertussis antigen of Pillemer produced both responses. The production of early immunity by pertussis vaccine was non-specific, for Dubos has shown that marked protection detectable within a few hours was produced in mice against staphylococcus and tubercle bacilli. This early immunity is probably not due to an increase in properdin, for it has been shown that mice given properdin at the time of challenge were no more resistant than normal mice.

\section{STEROIDS IN PHARMACY AND MEDICINE}

$T$

'HE chairman's address to the British Pharmaceutical Congress held at Bristol during September 2-6 was given by Mr. Frank Hartley. In it, he surveyed the broad field of the steroids, beginning with the earliest known members of the group, the sterols, and ending with some of the latest researches on anabolic steroids.

He referred briefly to cholesterol, the bile acids and antirachitic vitamins, mentioning some of their significance for pharmacy. He then directed his attention to the steroid hormones, dealing in turn with cestrogens, progestational substances, adrenocortical hormones, steroids as anæsthetics, androgens and anabolic agents. It is difficult to select, from this masterful survey, a few topics for special comment, and what follows must necessarily reflect my particular interest.

Mr. Hartley discussed some of the recent develop. ments in the search for clinically useful steroids among the progestational substances, adrenocortical hormones, androgens and anabolic substances. The 19-nor-steroids came in for a good deal of attention under several of these headings. 19-Norprogesterone is more active than progesterone, as is 19-norethisterone than ethisterone. 17 $\alpha$-Methyl-19nortestosterone (methylœstrenolone) is among the more potent of the new oral progestational substances; other derivatives, such as 17 1 -ethyl-19.

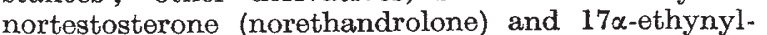
$5(10)$-œstrenolone, also exhibit progestational activity. Some of these compounds have marked anabolic properties as well.

Mr. Hartley mentioned the $\Delta^{1}$ derivatives of cortisone and hydrocortisone, the now well-known 
substances prednisone and prednisolone, and then discussed still more recent developments. An unsaturated linkage between $\mathrm{C}_{6}$ and $\mathrm{C}_{7}$ converts prednisone and prednisolone into even more potent glucocorticoids. Halogenation at $\mathrm{C}_{9}$ and methylation at $\mathrm{C}_{6}$ have yielded $9 \alpha$-fluoro- $6 \alpha$-methylprednisolone, which is stated to have a glucocorticoid activity some two hundred times that of hydrocortisone. $9 \alpha$-Fluorohydrocortisone is now well known for its potent mineralocorticoid activity. Mr. Hartley referred to numerous other derivatives which have been produced by the steroid chemists, including steroids with additional hydroxyl groups at carbons $2,5,12,14$ and 16 . The potential value of these remains to be explored. The 19-nor analogues of cortisone and hydrocortisone are less potent than the parent substances.

Mention has already been made of studies on 19-nortestosterone and its derivatives as anabolic agents; esterification with benzoic, cyclopentylpropionic or phenylpropionic acids prolongs their effectiveness by intramuscular injection. Recent work has shown that 4-chlorination or 6-methylation of testosterone derivatives increases their anabolic/ androgenic ratio. 'The androgenic activity of $11 \beta: 17 \beta$ dihydroxy - $9 \alpha$-fluoro $-17 \alpha$-methylandrost - 4 - en - 3 - one (fluoxymestrone) is stated to be ten times greater than that of methyltestosterone, but in spite of the presence of the 11-hydroxy group, it is said not to give rise to odema or to hypertension.

In concluding, Mr. Hartley pointed out that every natural steroid hormone possesses not one but a whole range of biological activities, some of them unnecessary or even undesirable in their application therapeutically. Consequently, the need has arisen for steroids 'tailored' to a particular therapeutic requirement. Progress towards meeting this need has been mentioned above. Some of the substances thus artificially created are often many times more active than the natural substances they replace. For the future, Mr. Hartley felt that the most important application of steroids would be in the field of gerontology, neuroendocrinology and population control. This progress will be achieved by the continued close collaboration of steroid chemists, biologists, pharmacists and elinicians.

G. I. M. SWYER

\section{RADIO RESEARCH 1956}

$\mathrm{T}$

HE annual reports of the chairman of the Radio Research Board and of the director of radio research are contained in the publication "Radio Research 1956"*. With the completion and occupation of the new buildings at the Radio Research Station, Slough, in the late summer of 1956 (see Nature, 180,$163 ; 1957)$, the final transfer of the former Radio Division of the National Physical Laboratory at Teddington to Slough has now been completed. In addition to the work at the main. Station at Slough, ionospheric observatories have continued to be maintained at Inverness, Port Stanley and Singapore, and further work is carried out under contract with the Department at the Universities of Cambridge, London and Wales. An ionospheric station has also been equipped and

* Department of Scientific and Industrial Research. Radio Research 1956 : The Report of the Radio Research Board and the Report of the Director of Radio Research.
(London: H.M. Stationery Office.) staffed at the Royal Society base at Halley Bay, Antarctica, in connexion with the International Geophysical Year.

The main emphasis of the research programme has been concerned with ionospheric and tropospheric radio-wave propagation over a very wide frequency band. The efficient forecasting of ionospheric transmission conditions is an important practical task, which has continued to be the concern of the Department. In addition to the routine ionospheric recordings made at the Department's observatories, studies of long-distance communications in the highfrequency band (17-24 Mc./s.) have been made on fixed frequencies, using signals from distant pulse transmitters. A variable-frequency sounding appar. atus, which operates in increments of $10 \mathrm{kc} / \mathrm{s}$. every 0.4 sec. in the frequency band $5 \cdot 5-25 \mathrm{Mc} / \mathrm{s}$., has also been constructed for studying long-distance propagation and given preliminary trials. Another aid to this study, which is now being pursued from the practical point of view, is the technique of backscatter sounding. In this, conditions for transmission over long distances are investigated by a radar method in which the signals are returned by backscatter at the ground, obviating the need for a distant transmitter. Good results have been obtained, and an operational equipment has now been built for routine use.

The study of forward-scatter in the ionosphere ( $E$-region) of signals in the very-high-frequency band $(>30 \mathrm{Mc} . / \mathrm{s}$.) has continued. Measurements of both the amplitude and the angular spread and direction of arrival of the scattered energy have been made in an attempt to elucidate the mechanism responsible. Definite conclusions are not yet possible, but it appears that the night-time signals are more likely to arise from weak reflexions from a large number of trails of ionization produced by low-magnitude meteors, rather than from scattering from turbulent irregularities in the ionosphere. In this work, strong steady signals are frequently received in the summer, due to direct reflexions from sporadic $E$-ionization. Investigations of the forward-scatter in the troposphere of very-high and ultra-high-frequencies have also been started at Slough, in collaboration with the Post Office. Experiments on a frequency of $1,370 \mathrm{Mc} / \mathrm{s}$. received over a path of $150 \mathrm{~km}$. are in progress, with the object of elucidating the propagation process and developing practical communication links based on the phenomenon. The study of the statistical correlation between certain meteorological features and field-strength measurements beyond the horizon of the transmitter has continued, as have also the effects of radio noise due to atmospherics on radio communications.

The possibilities of using very low frequencies (10-20 ke./s.) for long-distance navigational aids are being investigated in co-operation with the Admiralty. It is intended to develop a system which functions on the phase-difference between signals received from two or more widely separated phase-locked transmitters, and preliminary investigations of the variable phase changes introduced by the ionosphere are in progress.

Apart from a summary of the many studies of radio wave propagation undertaken by the Department, "Radio Research 1956" includes a brief description of some of the work sponsored at universities, and of theoretical work on the characteristics of the ionosphere. A particular aspect of the latter is a 